\title{
EDITORIAL
}

\section{Changing Pattern of Gastric Surgery}

Though it has been said for pong period that Peptic Ulcer disease may occur in any age group irrespective of sex, it has been proclaimed that this is the commonest affection of Stomach and Duodenum. Surgery related to peptic ulcer disease is mainly based on Combating the short term and long term complications of such. Surgeons were busy with managing the three Life threatening complications like perforation, haematemesis and gastric outlet obstruction. The epidemiology of peptic ulcer disease reflects the prevalence of $H$ pylori infection in different populations. The timely discovery of $\mathrm{H} 2$ blocker agents and Proton pump inhibitor made it possible to treat the most of the disease effectively by non surgical means. Later on , the concentration on eradication of $H$ pylori by medical means make it easier for the surgeon to manage the other diseases of stomach and so on.

Carcinoma of the stomach and other malignant diseases of the stomach clearly prevails in middle aged to elderly persons. It was one of the life threatening disease process from ancient and still prevails. Geographical distribution of malignant disease of the stomach is a great concern and the peoples resides in and around Japan are the worst sufferers. On the contrary Japanese management protocol based on "Tier" removal made it gold standard for rest of the world.

As the affluence persons of the world are increasing day by day and many of them are suffering from Obesity related problem, there are increasing concern on weight reduction by surgical means. Earlier days most of this surgery were open procedure and significant number of morbidity and mortality in relation to those made those un popular for the common people.

\section{Surgical treatment for Stomach Diseases}

There are a varieties of surgery for benign disease of the stomach. For repair of peptic ulcer perforation, most of the procedures are direct repair of the stomach. In addition lower partial resection, antrectomy, Emergency vagotomy-gastrojejunostomy are rarely performed. For haematemesis and melaena most of the patient are treated by non surgical means and endoscopic coagulation of blood vessel. However in case of deemed necessary, open lower partial gastrectomy or under running of bleeding vessel is a therapeutic choice. For Gastric outlet obstruction following scarred Duedenal or Pyloric ulcer a bypass procedure is practiced throughout the world, still today. A time tested procedure of Bilateral truncal vagotomy and gastrojejunostomy is still the choice of the surgeons mostly. A modification of selective or highly selective vagotomy or pylorus sparing vagotomy is also been popular in some parts of the world. Gastro-jejunostomy can be modified by pyloroplasty or antrectomy in some cases as well.

Surgery for gastric cancer remarkably changed based on its clear idea of knowledge of lymphatic distribution. The lymph nodes draining the stomach has a clear understamding and Contrast enhanced CT scan or MR scan made it possible to clearly define the lymphatic drainage of the stomach. Its based on tier like distribution and maping of these lymph nodes made it possible to predict the clearance of the local metastasis.

Laparoscopic procedure in stomach has revolutionalized the approach to surgery easier, better and safe handling thereafter. In addition discovery of endostapler and endovascular control made it more soft handling and a better outcome.

A small modification of palliative surgery for stomach is insertion PEJ catheter for advanced malignancy and also patients having prolonged parenteral nutrition. It is a combined procedure for surgeons and gastroenterologist as a team and in same sitting to insert the catheter safely. Generation of changes in Gastric Surgery

First generation:-1. Repair of perforation

1. Gagotomy- gastrojejunostomy

2. Antrectomy for haematemesis.

A. Second Generation:-1. Lower partial gastrectomy

1. Total Gastrectomy.

2. Roux-n- Y anastomoses.

3. Trans thoracic gastrectomy 
B. Third Generation:-1. Vertical Banded gstroplasty

1. Radical gastric dissection

2. PEJ insertion.

C. Fourth generation:-1. Laparoscopic Gastroplasty

1. Robotic assisted gastroplasty.

Recent studies demonstrated increasing feasibility for non-surgical palliation of unresectable gastrointestinal cancers as well as gastric outlet obstruction.1 Diagnostic laparoscopy was introduced as the final staging investigation in GI cancer patients who do not have advanced disease after radiological staging and therefore seem candidates for surgical resection. The aim of Diagnostic laparoscopy is to detect peritoneal, superficial liver, or lymph node metastasis and locally advanced disease those may be missed on radiological staging and thus could avoid a non- therapeutic laparotomy.2

The obesity epidemic and its associated diseases threaten to over burden global healthcare resources. Bariatric surgery is the only intervention that gives long standing improved or resolution of obesity related conditions and also survival benefits. It is highly cost effective. In the US and some European countries, the number of operations performed has now surpassed cholecystectomy.3

The three commonly performed procedures for morbid obesity are laparoscopic Roux-en-Y gastric bypass, laparoscopic adjustable gastric band and sleeve gastrectomy. 4

(J Banagladesh Coll Phys Surg 2015; 33: 118-119)

\section{Prof. M.A. Hashem Bhuiyan}

Professor \& Head

Dept. of Surgery

Dhaka Medical College \& Hospital

\section{References:}

1. Mittal A, Windsor I, Woodfield $\mathrm{J}$ et al. Matched study of three methods for palliation of malignant pyloroduodenal obstruction. Br J Surg2004; 91: 205-209.

2. Boyd JR, Nond HIJ. Diagnostic Laparoscopy: Endoscopy 2000. ; 32 : 153-158.

3. JostromL, Nasbro K, Sjostrom CD et al. Effects of bariatric surgery on mortality in Swedish obese patients. N Eng J Med 2007; 357: 741-52.

4. Quit Jl, Picot J et al. Surgery for obesity. Cochrane database syst review 2009; (2) CD 003641. 